\title{
Monolingual and Cross-Lingual Information Retrieval Models Based on (Bilingual) Word Embeddings
}

Ivan Vulić and Marie-Francine Moens

KU Leuven

Department of Computer Science

KU LEUVEN

ivan.vulic@cs.kuleuven. be

marie.francine.moens@cs . kuleuven . be

SIGIR 2015, Santiago de Chile; August 11, 2015 


\section{Presentation Plan I}

\section{Learning Bilingual Word Embeddings (BWEs)}

- Dense word representations, word embeddings, bilingual word embeddings

- Monolingual and bilingual embedding spaces

- Multilingual text data $\rightarrow$ why document-aligned data?

- New BWE learning model: BWESG $\rightarrow$ learning monolingual and bilingual embedding spaces 


\section{Presentation Plan II}

\section{BWEs in IR}

- Semantically-aware representations in the ad-hoc retrieval process?

- From word representations to query and document representations

- Monolingual embeddings $\rightarrow$ monolingual retrieval; Bilingual embeddings $\rightarrow$ cross-lingual retrieval

- The same conceptual model of retrieval for MoIR and CLIR with bilingual embeddings spaces!

- Results and discussion 
Part I: Learning BWEs 


\section{Learning Word Representations}

\section{Key idea}

Distributional hypothesis $\rightarrow$ words with similar meanings are likely to appear in similar contexts

[Harris, Word 1954]

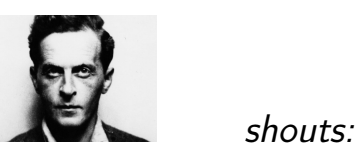

"Meaning as use!"

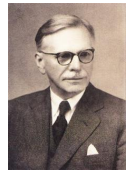

calmly states:

"You shall know a word by the company it keeps." 


\section{Word Embeddings}

Dense representations $\rightarrow$ real-valued low-dimensional vectors (seen already? LSI?)

Word embedding induction

$\rightarrow$ learn word-level features which generalize well across tasks and languages

$\rightarrow$ bilingual word embeddings (this talk)

Word embeddings capture interesting and universal features:

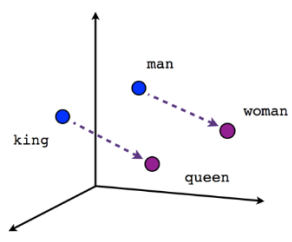

Male-Female

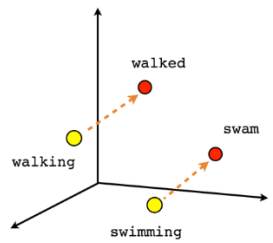

Verb tense

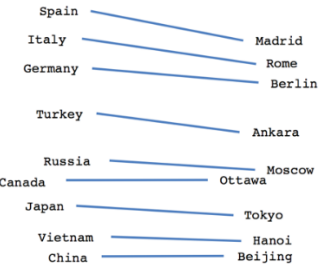

Country-Capital 


\section{Embedding Spaces $=$ Semantic Spaces}

3D embedding

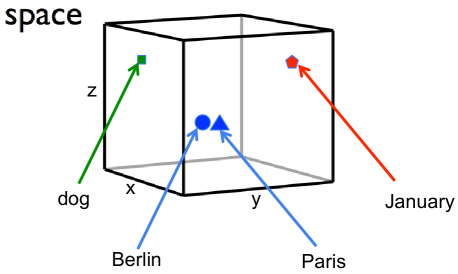

Monolingual
3D embedding

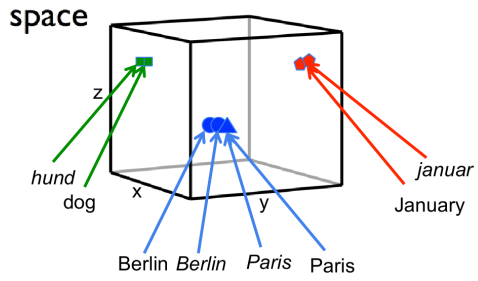

Bilingual

[Image courtesy of Stephan Gouws] 


\section{Bilingual Word Embeddings}

Representation of a word $w_{1}^{S} \in V^{S}$ :

$$
\operatorname{vec}\left(w_{1}^{S}\right)=\left[f_{1}^{1}, f_{2}^{1}, \ldots, f_{\text {dim }}^{1}\right]
$$

Exactly the same representation for $w_{2}^{T} \in V^{T}$ :

$$
\operatorname{vec}\left(w_{2}^{T}\right)=\left[f_{1}^{2}, f_{2}^{2}, \ldots, f_{\text {dim }}^{2}\right]
$$

Language-independent word representations in the same shared semantic (or embedding) space!

Word representation $\rightarrow \mathbf{A}$ dense real-valued dim-dimensional vector, these dimensions are no longer interpretable (unlike with other semantic representations). 


\section{Back to Monolingual...}

Skip-gram with negative sampling (SGNS)

[Mikolov et al.: NIPS 2013]

INPUT PROJECTION OUTPUT

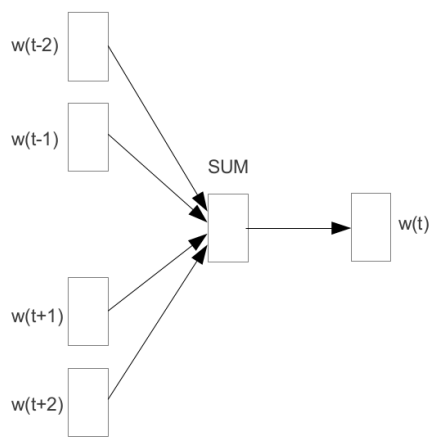

CBOW
INPUT PROJECTION OUTPUT

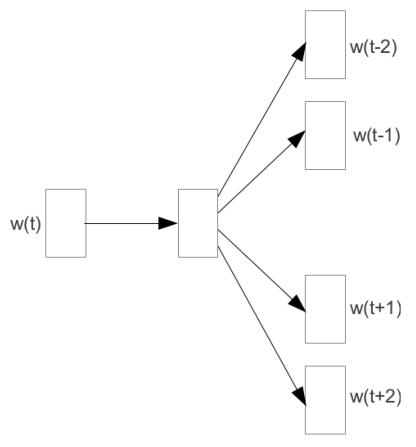

Skip-gram 


\section{Back to Monolingual...}

Skip-gram with negative sampling (SGNS)

[Mikolov et al.; NIPS 2013]

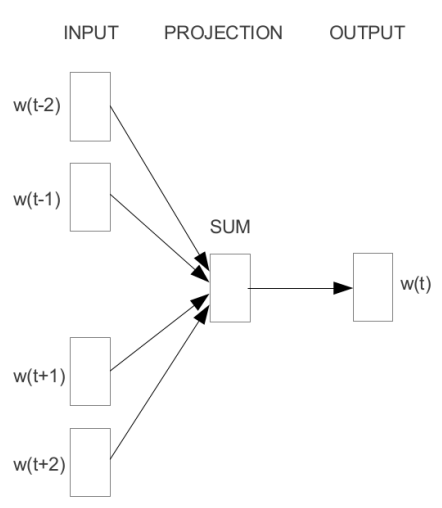

CBOW

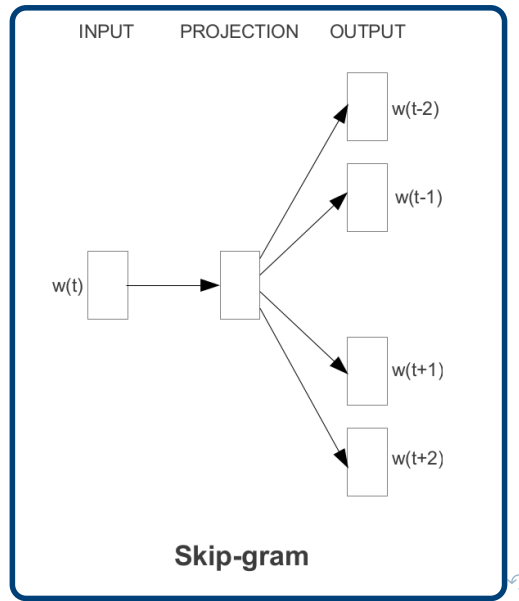




\section{Back to Monolingual...}

\section{Skip-gram with negative sampling (SGNS)}

[Mikolov et al.; NIPS 2013]

Learning from the set $D$ of (word, context) pairs observed in a corpus: $(w, v)=(w(t), w(t \pm i)) ; i=1, \ldots, c s ; c s=$ context window size

SG learns to predict the context of the pivot word

John saw a cute gray huhblub running in the field.

$D=$ (huhblub, cute), (huhblub, gray), (huhblub, running), (huhblub, in) $\operatorname{vec}($ huhblub $)=[-0.23,0.44,-0.76,0.33,0.19, \ldots]$ 


\section{Back to Monolingual...}

Negative sampling = learning using both positive ("observed") examples (set $D$ ), and negative ("unobserved") examples (set $D^{\prime}$ )

SGNS is actually doing something very similar to the older approaches $\rightarrow$ factorizing the traditional word-context matrix! [Levy et al., NIPS 2014, TACL 2015]

More research focused on learning monolingual WEs:

- Full-fledged neural-net approaches [Bengio et al., JMLR 2003; Collobert and Weston, ICML 2008]

- Other factorization methods (e.g., Hellinger PCA) [Lebret and Collobert, EACL 2014]

- GloVe [Pennington et al., EMNLP 2014]

- ... 


\section{SGNS - A More Formal Summary}

Probability for one word-context pair $(w, v)$ :

$$
P(D=1 \mid w, v, \theta)=\frac{1}{1+\exp \left(-\vec{w} \cdot \overrightarrow{v_{c}}\right)}
$$

General objective:

$$
J=\arg \max _{\theta} \sum_{(w, v) \in D} \log \frac{1}{1+\exp \left(-\vec{w} \cdot \overrightarrow{v_{c}}\right)}
$$

General objective with negative sampling:

$$
J=\arg \max _{\theta} \sum_{(w, v) \in D} \log \frac{1}{1+\exp \left(-\vec{w} \cdot \overrightarrow{v_{c}}\right)}+\sum_{\left(w, v^{\prime}\right) \in D^{\prime}} \log \frac{1}{1+\exp \left(\vec{w} \cdot \overrightarrow{v_{c}^{\prime}}\right)}
$$

[Goldberg and Levy: word2vec explained; arXiv 2014] 


\section{And Now Back to Bilingual...}

Generalizing the WE learning in bilingual settings using the similar principles...

$3 \mathrm{D}$ embedding

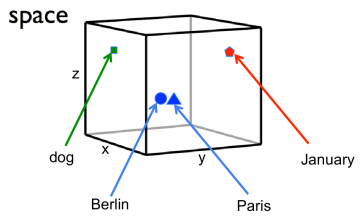

3D embedding

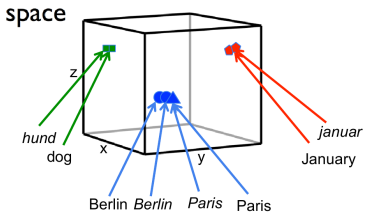

1. Align pretrained monolingual embedding spaces (offline) using dictionaries [Mikolov et al., arXiv 2013; Lazaridou et al., ACL 2015]

2. Jointly learn and align embeddings (online) using parallel-only data [Hermann and Blunsom, ACL 2014; Chandar et al., NIPS 2014] 3. Jointly learn and align embeddings (online) using mono and parallel data [Gouws et al., ICML 2015; Soyer et al., ICLR 2015, Shi et al., ACL 2015] 


\section{And Now Back to Bilingual...}

Generalizing the WE learning in bilingual settings using the similar principles...

3D embedding

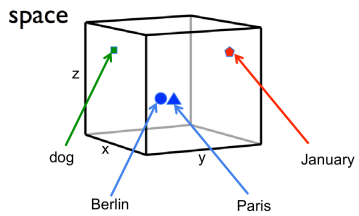

3D embedding

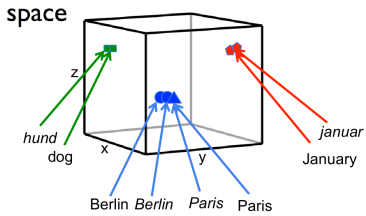

1. Align pretrained monolingual embedding spaces (offline) using dictionaries [Mikolov et al., arXiv 2013; Lazaridou et al., ACL 2015]

2. Jointly learn and align embeddings (online) using parallel-only data [Hermann and Blunsom, ACL 2014; Chandar et al., NIPS 2014]

3. Jointly learn and align embeddings (online) using mono and parallel data [Gouws et al., ICML 2015; Soyer et al., ICLR 2015, Shi et al., ACL 2015]

4. Can we do it without readily available dictionaries and parallel data? $\rightarrow$ Using document-aligned data (e.g., Wikipedia) [our model: BWESG] 


\section{BWEs from Document-Aligned Data}

Input: Pivot word representation

Output: Context representations

Aligned document pair

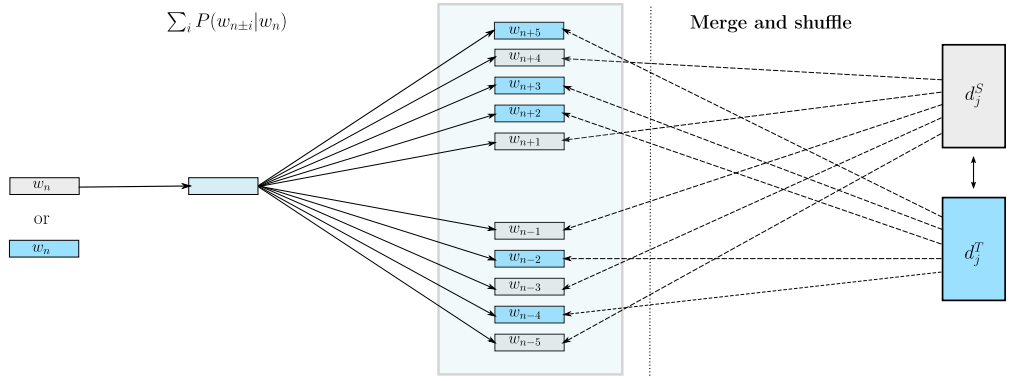




\section{BWEs from Document-Aligned Data}

Input: Pivot word representation

Output: Context representations

Aligned document pair

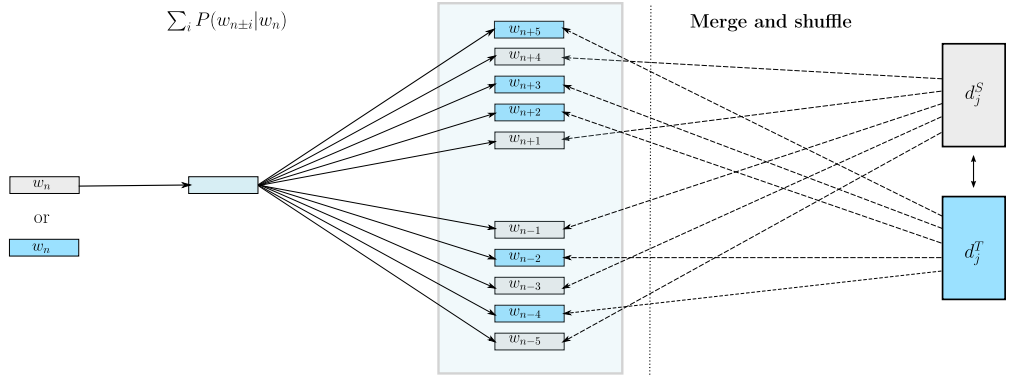

$\rightarrow$ Merge \& Shuffle: Training a SGNS (or any other monolingual model!) on shuffled "pseudo-bilingual" documents $\rightarrow$

$\rightarrow$ Our model: BWESG 


\section{BWEs from Document-Aligned Data}

Input: Pivot word representation

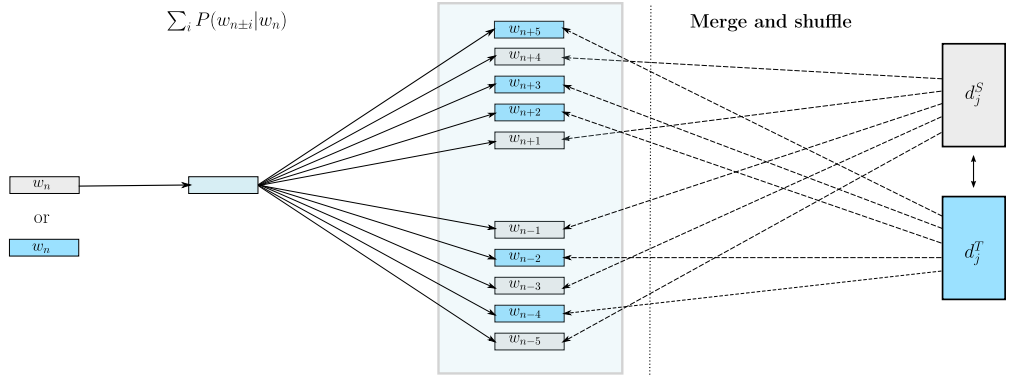

$\rightarrow$ Merge \& Shuffle: Training a SGNS (or any other monolingual model!) on shuffled "pseudo-bilingual" documents $\rightarrow$

$\rightarrow$ Our model: BWESG

$\rightarrow$ 1. dumb shuffling: random (this work); 2. slightly more intelligent: length ratio-based (after this work); 3. even more intelligent: future work 


\section{BWEs from Document-Aligned Data}

Input: Pivot word representation

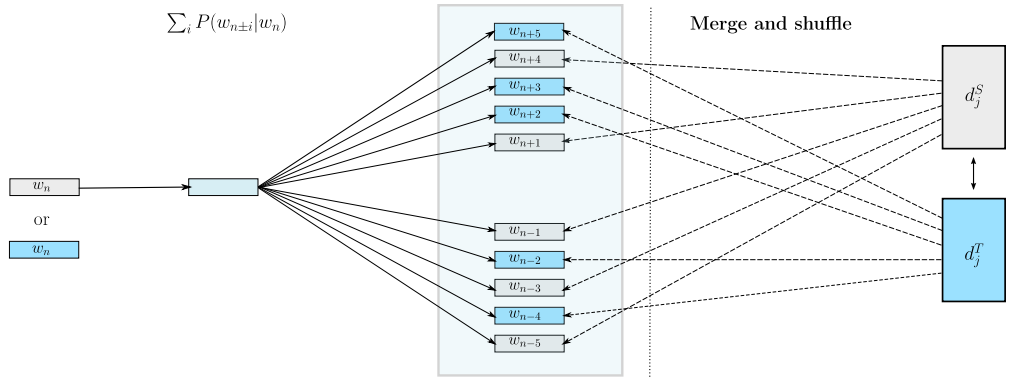

$\rightarrow$ shuffling ensures bilingual (instead of monolingual) contexts $\rightarrow$ learning a bilingual embedding space jointly (online) 


\section{BWEs from Document-Aligned Data}

Input: Pivot word representation

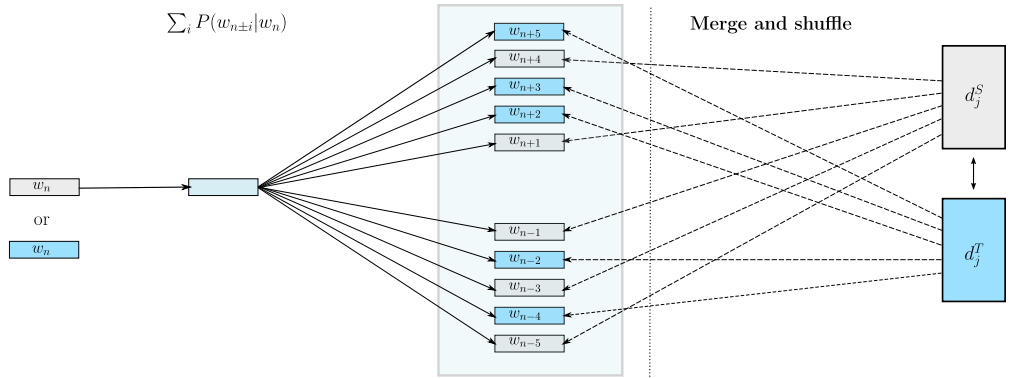

$\rightarrow$ shuffling ensures bilingual (instead of monolingual) contexts $\rightarrow$ learning a bilingual embedding space jointly (online)

$\rightarrow$ No longer a local model: Window size controls the number of document-level positive samples 


\section{BWEs with BWESG - Examples}

\begin{tabular}{|c|c|c|c|c|c|c|c|c|}
\hline \multicolumn{3}{|c|}{ Spanish-English (ES-EN) } & \multicolumn{3}{|c|}{ Italian-English (IT-EN) } & \multicolumn{3}{|c|}{ Dutch-English (NL-EN) } \\
\hline $\begin{array}{l}(1) \\
\text { reina }\end{array}$ & $\begin{array}{l}(2) \\
\text { reina }\end{array}$ & $\begin{array}{l}(3) \\
\text { reina }\end{array}$ & $\begin{array}{l}(1) \\
\text { madre }\end{array}$ & $\begin{array}{l}(2) \\
\text { madre }\end{array}$ & $\begin{array}{l}(3) \\
\text { madre }\end{array}$ & $\begin{array}{l}\text { (1) } \\
\text { schilder }\end{array}$ & $\begin{array}{l}(2) \\
\text { schilder }\end{array}$ & $\begin{array}{l}\text { (3) } \\
\text { schilder }\end{array}$ \\
\hline (Spanish) & (English) & (Combined) & (Italian) & (English) & (Combined) & (Dutch) & (English) & (Combined) \\
\hline $\begin{array}{l}\text { rey } \\
\text { trono } \\
\text { monarca } \\
\text { heredero } \\
\text { matrimonio } \\
\text { hijo } \\
\text { reino } \\
\text { reinado } \\
\text { regencia } \\
\text { duque }\end{array}$ & $\begin{array}{l}\text { queen }(+) \\
\text { heir } \\
\text { throne } \\
\text { king } \\
\text { royal } \\
\text { reign } \\
\text { succession } \\
\text { princess } \\
\text { marriage } \\
\text { prince }\end{array}$ & $\begin{array}{l}\text { queen }(+) \\
\text { rey } \\
\text { trono } \\
\text { heir } \\
\text { throne } \\
\text { monarca } \\
\text { heredero } \\
\text { king } \\
\text { matrimonip } \\
\text { royal }\end{array}$ & $\begin{array}{l}\text { padre } \\
\text { moglie } \\
\text { sorella } \\
\text { figlia } \\
\text { figlio } \\
\text { fratello } \\
\text { casa } \\
\text { amico } \\
\text { marito } \\
\text { donna }\end{array}$ & $\begin{array}{l}\text { mother }(+) \\
\text { father } \\
\text { sister } \\
\text { wife } \\
\text { daughter } \\
\text { son } \\
\text { friend } \\
\text { childhood } \\
\text { family } \\
\text { cousin }\end{array}$ & $\begin{array}{l}\text { mother }(+) \\
\text { padre } \\
\text { moglie } \\
\text { father } \\
\text { sorella } \\
\text { figlia } \\
\text { figlio } \\
\text { sister } \\
\text { fratello } \\
\text { wife }\end{array}$ & $\begin{array}{l}\text { kunstschild } \\
\text { schilderij } \\
\text { kunstenaar } \\
\text { olieverf } \\
\text { olieverfschil } \\
\text { schilderen } \\
\text { frans } \\
\text { nederlands } \\
\text { componist } \\
\text { beeldhouwe }\end{array}$ & $\begin{array}{l}\text { epainter(+) } \\
\text { painting } \\
\text { portrait } \\
\text { artist } \\
\text { Ideaijvas } \\
\text { impressionis } \\
\text { cubism } \\
\text { art } \\
\text { poet } \\
\text { rdrawing }\end{array}$ & $\begin{array}{l}\text { painter }(+) \\
\text { kunstschilder } \\
\text { painting } \\
\text { schilderij } \\
\text { kunstenaar } \\
\text { stportrait } \\
\text { olieverf } \\
\text { olieverfschilderij } \\
\text { schilderen } \\
\text { artist }\end{array}$ \\
\hline
\end{tabular}

$\overrightarrow{r e i n a}-\overrightarrow{w o m a n}+\overrightarrow{m a n} \approx \overrightarrow{r e y}$

$\overrightarrow{\text { queen }}-\overrightarrow{\text { mujer }}+\overrightarrow{\text { hombre }} \approx \overrightarrow{k i n g}$

$\overrightarrow{\text { reina }}-\overrightarrow{\text { mujer }}+\overrightarrow{\text { hombre }} \approx \overrightarrow{r e y}$ 


\section{Summary of Contributions}

- A novel model for learning bilingual word embeddings (BWEs) from non-parallel document-aligned data

- A simple framework for constructing query and document embeddings

- A unified framework for MolR and CLIR based on (bilingual) word embeddings 
Part II: BWEs in IR 


\section{Beyond the Level of Words}

We learn word embeddings:

$v e c($ huhblub $)=[-0.23,0.44,-0.76,0.33,0.19, \ldots]$

$\operatorname{vec}($ fluffy $)=[0.31,0.02,-0.11,-0.28,0.52, \ldots]$

$\rightarrow$ How to build document and query embeddings?

vec (huhblup is fluffy $)=$ ??

Adapting the framework from compositional distributional

semantics: [Mitchell and Lapata, ACL 2008; Socher et al., EMNLP 2011;

Milajevs et al., EMNLP 2014] and many more...

A generic composition with a bag-of-words assumption:

$\left(d=\left\{w_{1}, w_{2}, \ldots, w_{\left|N_{d}\right|}\right\}\right)$

$$
\vec{d}=\overrightarrow{w_{1}} \star \overrightarrow{w_{2}} \star \ldots \star \overrightarrow{w_{\left|N_{d}\right|}}
$$

$\star=$ compositional vector operator (addition, multiplication, tensor product,.. ) 


\section{Document and Query Embeddings}

A general framework $\rightarrow$ in this work the simple and effective additive composition:

[Mitchell and Lapata, ACL 2008]

$$
\vec{d}=\overrightarrow{w_{1}}+\overrightarrow{w_{2}}+\ldots+\overrightarrow{w_{\left|N_{d}\right|}}
$$

The dim-dimensional document embedding in the same bilingual word embedding space:

$$
\vec{d}=\left[f_{d, 1}, \ldots, f_{d, k}, \ldots, f_{d, d i m}\right]
$$

$\rightarrow$ the ADD-BASIC composition model 


\section{Document and Query Embeddings}

A slightly more intelligent idea $\rightarrow$ weighting the summands using their self information computed in the target collection:

$$
s i_{w}=-\ln \frac{\operatorname{freq}(w, \mathcal{D C})}{\left|N_{\mathcal{D C}}\right|}
$$

freq $(w, \mathcal{D C})=$ frequency of $w$ in the collection

A SI-weighted sum:

$$
\vec{d}=s i_{w_{1}} \cdot \overrightarrow{w_{1}}+s i_{w_{2}} \cdot \overrightarrow{w_{2}}+\ldots+s i_{w_{\left|N_{d}\right|}} \cdot \overrightarrow{w_{\left|N_{d}\right|}}
$$

$\rightarrow$ the ADD-SI composition model 


\section{Document and Query Embeddings}

$\rightarrow$ The same principles with queries

$\rightarrow$ Using only ADD-BASIC

$$
\vec{Q}=\overrightarrow{q_{1}}+\overrightarrow{q_{2}}+\ldots+\overrightarrow{q_{m}}
$$

The dim-dimensional query embedding in the same bilingual word embedding space:

$$
\vec{Q}=\left[f_{Q, 1}, \ldots, f_{Q, k}, \ldots, f_{Q, d i m}\right]
$$




\section{Final BWE-Based MoIR and CLIR I}

(1) Induce a bilingual word embedding space using any BWE induction model $\rightarrow$ in this work: BWESG

(2) Given is a target document collection $\mathcal{D C}=\left\{d_{1}^{\prime}, \ldots, d_{N^{\prime}}^{\prime}\right\}$.

Compute dim-dimensional document embeddings $\overrightarrow{d^{\prime}}$ for each $d^{\prime} \in \mathcal{D C}$ using the dim-dimensional WEs from the set $\mathcal{B W} \mathcal{E}$ obtained in the previous step and a semantic composition model (ADD-BASIC or ADD-SI something anything else).

(3) After the query $Q=\left\{q_{1}, \ldots, q_{m}\right\}$ is issued in language $L_{S}$, compute a dim-dimensional query embedding using the ADD-BASIC composition model. 


\section{Final BWE-Based MoIR and CLIR II}

(4) For each $d^{\prime} \in \mathcal{D C}$, compute the semantic similarity score $\operatorname{sim}\left(d^{\prime}, Q\right)$ which quantifies each document's relevance to the query $Q$ :

$$
\operatorname{sim}\left(d^{\prime}, Q\right)=S F\left(d^{\prime}, Q\right)=\frac{\overrightarrow{d^{\prime}} \cdot \vec{Q}}{\left|\overrightarrow{d^{\prime}}\right| \cdot|\vec{Q}|}
$$

(6) Rank all documents from $\mathcal{D C}$ according to their similarity scores from the previous step.

WE-VS: WE-based MoIR and CLIR models (using ADD-BASIC) 
Part IIb: Experiments 


\section{BWESG Training Setup}

- Stochastic gradient descent with a default global learning rate 0.025

- Other default word2vec parameters: subsampling rate $1 e-4$, negative sampling with 25 negative samples, 15 epochs

- 10 random corpora shuffles, although we advocate the use of a more intelligent shuffling procedure (developed after the paper was released)

- $d=100-800$ in steps of 100

- $c s=10-100$ in steps of 10 


\section{Training Collections}

\section{[English|Dutch] $\rightarrow$ [English|Dutch] retrieval}

Exactly the same setup as in: [Vulić et al., Information Retrieval 2013, ECIR 2013]

Training data Europarl 6,206 documents (parallel) Wikipedia 7,612 documents (comparable)

\section{Vocabulary size English 76, 555 words} Dutch 71,168 words

$\rightarrow$ Stop words removed

$\rightarrow$ We exploit document-level alignments as the only bilingual signal (even for Europarl) 


\section{Test Collections}

[English|Dutch] $\rightarrow$ [English|Dutch] retrieval (using CLEF 2001-2003 campaigns)

\section{Monolingual}

\begin{tabular}{lllll}
\hline Direction & DC & \# Docs & Query Set & \# Queries \\
\hline EN $\rightarrow$ EN 2001 & LAT & 110,861 & EN'01: $41-90$ & 47 \\
EN $\rightarrow$ EN 2002 & LAT & 110,861 & EN'02: $91-140$ & 42 \\
EN $\rightarrow$ EN 2003 & LAT+GH & 166,753 & EN'03: 141-200 & 53 \\
\hline$N L \rightarrow$ NL 2001 & NC+AD & 190,604 & NL'01: $41-90$ & 50 \\
$N L \rightarrow$ NL 2002 & NC+AD & 190,604 & NL'02: $91-140$ & 50 \\
$N L \rightarrow$ NL 2003 & NC+AD & 190,604 & NL'03: 141-200 & 56 \\
\hline
\end{tabular}




\section{Test Collections}

\section{[English|Dutch] $\rightarrow$ [English|Dutch] retrieval (using CLEF 2001-2003 campaigns)}

\section{Cross-lingual}

\begin{tabular}{lllll}
\hline Direction & DC & \# Docs & Query Set & \# Queries \\
\hline $\mathrm{NL} \rightarrow$ EN 2001 & LAT & 110,861 & NL'01: $41-90$ & 47 \\
$\mathrm{NL} \rightarrow$ EN 2002 & LAT & 110,861 & NL'01: $91-140$ & 42 \\
$\mathrm{NL} \rightarrow$ EN 2003 & LAT+GH & 166,753 & NL'03: 141-200 & 53 \\
\hline $\mathrm{EN} \rightarrow$ NL 2001 & NC+AD & 190,604 & EN'01: $41-90$ & 50 \\
$\mathrm{EN} \rightarrow$ NL 2002 & NC+AD & 190,604 & EN'02: $91-140$ & 50 \\
EN $\rightarrow$ NL 2003 & NC+AD & 190,604 & EN'03: 141-200 & 56 \\
\hline
\end{tabular}

$\rightarrow$ Queries extracted from the title + description fields

$\rightarrow$ Stop words removed $\rightarrow$ Measuring MAP 


\section{Models in Comparison}

Single models:

1. WE-VS: Our WE-based retrieval model

2. LM-UNI: Unigram query likelihood language model with standard Dirichlet smoothing

3. LDA-IR: Semantically-aware (Bi)LDA-based QL model

[Wei and Croft, SIGIR 2006; Vulić et al, IR 2013]

A detailed description of all the models along with their parameter setup in the paper! 


\section{Models in Comparison}

Combined models:

1. LM-UNI+LDA-IR: A linear combination of the two single models:

[Wei and Croft, SIGIR 2006; Vulić et al, IR 2013]

$$
P\left(q_{i} \mid d\right)=\lambda P_{l d a}\left(q_{i} \mid d\right)+(1-\lambda) P_{l m}\left(q_{i} \mid d\right)
$$

2. LM-UNI+WE-VS: A linear combination of LM-UNI and WE-VS (to directly compare the "quality of semantic awareness" in the retrieval process)

x. GT+LM+LDA (only for CLIR): Translating a query using Google Translate, and then employing LM-UNI+LDA-IR on the translated query

Again, a detailed description of all the models along with their parameter setup in the paper! 


\section{Results - MolR}

\begin{tabular}{|c|c|c|c|c|c|c|}
\hline \multirow[b]{2}{*}{ Model } & \multicolumn{3}{|c|}{$\mathrm{EN} \rightarrow \mathrm{EN}$} & \multicolumn{3}{|c|}{$\mathrm{NL} \rightarrow \mathrm{NL}$} \\
\hline & 2001 & 2002 & 2003 & 2001 & 2002 & 2003 \\
\hline LM-UNI & .381 & .360 & .359 & .256 & .323 & .357 \\
\hline $\begin{array}{l}\text { LDA-IR } \\
\text { dim:300;cs:60 }\end{array}$ & .279 & .216 & .241 & .131 & .143 & .130 \\
\hline $\begin{array}{l}\text { WE-VS } \\
\text { dim:600;cs:60 }\end{array}$ & $.324 x$ & $.258 x$ & $.257 y$ & $.203 x$ & $.237 x$ & $.224 x$ \\
\hline WE-VS & $.329 x$ & $.281 x$ & $.262 y$ & $.204 x$ & $.262 x$ & $.231 x$ \\
\hline $\begin{array}{l}\text { LM+LDA } \\
\text { dim:300;cs:60 }\end{array}$ & .399 & .360 & .379 & .260 & .326 & .357 \\
\hline LM+WE $(\lambda=0.3)$ & $.412 y$ & $.381 x$ & $.401 y$ & $.271 x$ & $.349 x$ & $.372 x$ \\
\hline LM+WE $(\lambda=0.5)$ & $.429 x$ & $.394 x$ & $.407 x$ & $.279 x$ & $.370 x$ & $.382 x$ \\
\hline $\begin{array}{l}\text { LM+WE }(\lambda=0.7) \\
\operatorname{dim}: 600 ; c s: 60\end{array}$ & $.451 x$ & $.392 y$ & .389 & .270 & $.364 x$ & $.373 y$ \\
\hline LM+WE $(\lambda=0.3)$ & $.419 y$ & $.382 x$ & $.403 y$ & $.274 x$ & $.350 x$ & $.373 x$ \\
\hline LM+WE $(\lambda=0.5)$ & $.436 x$ & $.391 x$ & $.408 x$ & $.282 x$ & $.371 x$ & $.383 x$ \\
\hline LM+WE $(\lambda=0.7)$ & $.430 x$ & $.392 y$ & .381 & .268 & $.367 x$ & $.374 y$ \\
\hline
\end{tabular}




\section{Results - MolR}

$$
\mathrm{EN} \rightarrow \mathrm{EN} \quad \mathrm{NL} \rightarrow \mathrm{NL}
$$

\begin{tabular}{lcccccc|}
\hline Model & 2001 & 2002 & 2003 & 2001 & 2002 & 2003 \\
\hline LM-UNI & .381 & .360 & .359 & .256 & .323 & .357 \\
\hline $\begin{array}{l}\text { LDA-IR } \\
\text { dim:300; cs:60 }\end{array}$ & .279 & .216 & .241 & .131 & .143 & .130 \\
WE-VS & $.324 x$ & $.258 x$ & $.257 y$ & $.203 x$ & $.237 x$ & $.224 x$ \\
dim:600; cs:60 & & & & & & \\
WE-VS & $.329 x$ & $.281 x$ & $.262 y$ & $.204 x$ & $.262 x$ & $.231 x$ \\
\hline LM+LDA & .399 & .360 & .379 & .260 & .326 & .357 \\
dim:300; $c s: 60$ & & & & & & \\
LM+WE $(\lambda=0.3)$ & $.412 y$ & $.381 x$ & $.401 y$ & $.271 x$ & $.349 x$ & $.372 x$ \\
LM+WE $(\lambda=0.5)$ & $.429 x$ & $.394 x$ & $.407 x$ & $.279 x$ & $.370 x$ & $.382 x$ \\
LM+WE $(\lambda=0.7)$ & $.451 x$ & $.392 y$ & .389 & .270 & $.364 x$ & $.373 y$ \\
dim:600; $c s: 60$ & & & & & & \\
LM+WE $(\lambda=0.3)$ & $.419 y$ & $.382 x$ & $.403 y$ & $.274 x$ & $.350 x$ & $.373 x$ \\
LM+WE $(\lambda=0.5)$ & $.436 x$ & $.391 x$ & $.408 x$ & $.282 x$ & $.371 x$ & $.383 x$ \\
LM+WE $(\lambda=0.7)$ & $.430 x$ & $.392 y$ & .381 & .268 & $.367 x$ & $.374 y$ \\
\hline
\end{tabular}




\section{Results - MolR}

\begin{tabular}{|c|c|c|c|c|c|c|}
\hline \multirow[b]{2}{*}{ Model } & \multicolumn{3}{|c|}{$\mathrm{EN} \rightarrow \mathrm{EN}$} & \multicolumn{3}{|c|}{$\mathrm{NL} \rightarrow \mathrm{NL}$} \\
\hline & 2001 & 2002 & 2003 & 2001 & 2002 & 2003 \\
\hline LM-UNI & .381 & .360 & .359 & .256 & .323 & .357 \\
\hline $\begin{array}{l}\text { LDA-IR } \\
\operatorname{dim}: 300 ; c s: 60\end{array}$ & .279 & .216 & .241 & .131 & .143 & .130 \\
\hline $\begin{array}{l}\text { WE-VS } \\
\text { dim:600; cs:60 }\end{array}$ & $.324 x$ & $.258 x$ & $.257 y$ & $.203 x$ & $.237 x$ & $.224 x$ \\
\hline WE-VS & $.329 x$ & $.281 x$ & $.262 y$ & $.204 x$ & $.262 x$ & $.231 x$ \\
\hline $\begin{array}{l}\text { LM+LDA } \\
\operatorname{dim}: 300 ; c s: 60\end{array}$ & .399 & .360 & .379 & .260 & .326 & .357 \\
\hline LM+WE $(\lambda=0.3)$ & $.412 y$ & $.381 x$ & $.401 y$ & $.271 x$ & $.349 x$ & $.372 x$ \\
\hline LM+WE $(\lambda=0.5)$ & $.429 x$ & $.394 x$ & $.407 x$ & $.279 x$ & $.370 x$ & $.382 x$ \\
\hline $\begin{array}{l}\text { LM+WE }(\lambda=0.7) \\
\operatorname{dim}: 600 ; c s: 60\end{array}$ & $.451 x$ & $.392 y$ & .389 & .270 & $.364 x$ & $.373 y$ \\
\hline LM+WE $(\lambda=0.3)$ & $.419 y$ & $.382 x$ & $.403 y$ & $.274 x$ & $.350 x$ & $.373 x$ \\
\hline LM+WE $(\lambda=0.5)$ & $.436 x$ & $.391 x$ & $.408 x$ & $.282 x$ & $.371 x$ & $.383 x$ \\
\hline LM+WE $(\lambda=0.7)$ & $.430 x$ & $.392 y$ & .381 & .268 & $.367 x$ & $.374 y$ \\
\hline
\end{tabular}




\section{Results - MolR II}

Testing the influence of dimensionality...

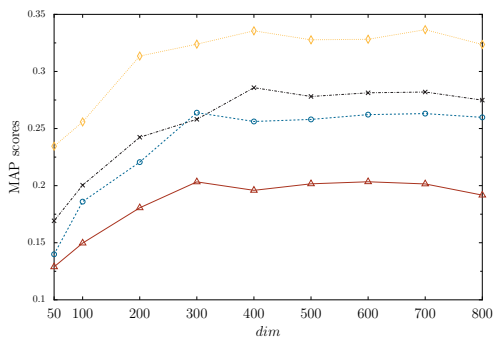

(a) WE-VS, $c s=60$

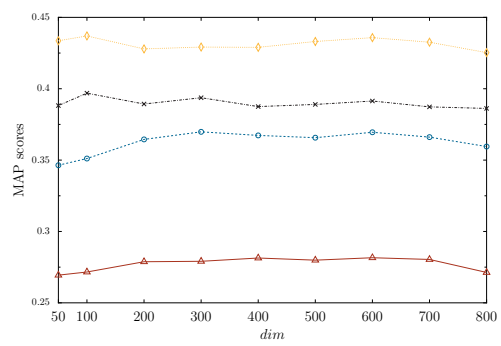

(b) LM-UNI+WE-VS, $c s=60$ 


\section{Results - MolR III}

..and window size... (controlling the data dropout)

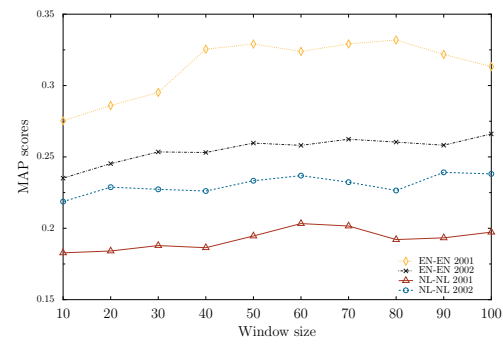

(c) WE-VS, $\operatorname{dim}=300$

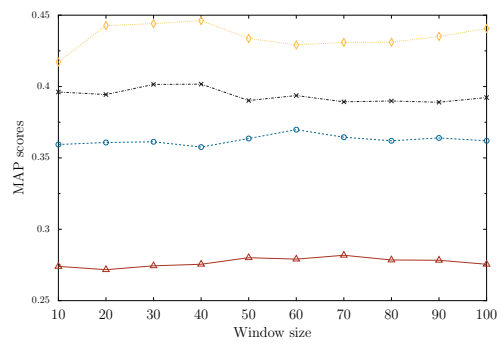

(d) LM-UNI+WE-VS, dim $=300$ 


\section{Results - CLIR}

\begin{tabular}{lccccccc}
\hline & \multicolumn{3}{l}{$\mathrm{NL} \rightarrow \mathrm{EN}$} & \multicolumn{3}{l}{$\mathrm{EN} \rightarrow \mathrm{NL}$} \\
\cline { 2 - 3 } \cline { 7 - 8 } Model & 2001 & 2002 & 2003 & & 2001 & 2002 & 2003 \\
\hline LM-UNI & .094 & .108 & .092 & .078 & .125 & .112 \\
\hline LDA-IR & .197 & .139 & .123 & .145 & .137 & .171 \\
dim:300; $c s: 60$ & & & & & & \\
WE-VS & .187 & $.204 x$ & .120 & .174 & $.185 y$ & .157 \\
dim:600; $c s: 60$ & & & & & & \\
WE-VS & $.222 y$ & $.230 x$ & .127 & $.178 y$ & $.219 x$ & .181 \\
\hline LM+LDA & .267 & .225 & .199 & .225 & .268 & .278 \\
GT+LM+LDA & .307 & .275 & .248 & .230 & .240 & .244 \\
dim:300; $c s: 60$ & & & & & & \\
LM+WE $(\lambda=0.3)$ & .189 & .273 & .197 & .101 & .159 & .150 \\
LM+WE $(\lambda=0.5)$ & .218 & $.283 y$ & .220 & .113 & .184 & .167 \\
LM+WE $(\lambda=0.7)$ & .255 & $.307 x$ & .219 & .180 & .209 & .208 \\
dim:600; $c s: 60$ & & & & & & \\
LM+WE $(\lambda=0.3)$ & .205 & $.281 y$ & .198 & .107 & .167 & .154 \\
LM+WE $(\lambda=0.5)$ & .236 & $.299 x$ & .215 & .123 & .203 & .183 \\
LM+WE $(\lambda=0.7)$ & .286 & $.317 x$ & .222 & .190 & .249 & .225 \\
\hline
\end{tabular}




\section{Results - CLIR}

\begin{tabular}{|c|c|c|c|c|c|c|}
\hline \multirow[b]{2}{*}{ Model } & \multicolumn{3}{|c|}{$\mathrm{NL} \rightarrow \mathrm{EN}$} & \multicolumn{3}{|c|}{$\mathrm{EN} \rightarrow \mathrm{NL}$} \\
\hline & 2001 & 2002 & 2003 & 2001 & 2002 & 2003 \\
\hline LM-UNI & .094 & .108 & .092 & .078 & .125 & .112 \\
\hline $\begin{array}{l}\text { LDA-IR } \\
\operatorname{dim}: 300 ; c s: 60\end{array}$ & .197 & .139 & .123 & .145 & .137 & .171 \\
\hline $\begin{array}{l}\text { WE-VS } \\
\operatorname{dim}: 600 ; c s: 60\end{array}$ & .187 & $.204 x$ & .120 & .174 & $.185 y$ & .157 \\
\hline WE-VS & $.222 y$ & $.230 x$ & .127 & $.178 y$ & $.219 x$ & .181 \\
\hline LM+LDA & .267 & .225 & .199 & .225 & .268 & .278 \\
\hline $\begin{array}{l}\mathrm{GT}+\mathrm{LM}+\mathrm{LDA} \\
\operatorname{dim}: 300 ; c s: 60\end{array}$ & .307 & .275 & .248 & .230 & .240 & .244 \\
\hline LM+WE $(\lambda=0.3)$ & .189 & .273 & .197 & .101 & .159 & .150 \\
\hline LM+WE $(\lambda=0.5)$ & .218 & $.283 y$ & .220 & .113 & .184 & .167 \\
\hline $\begin{array}{l}\text { LM+WE }(\lambda=0.7) \\
\operatorname{dim}: 600 ; c s: 60\end{array}$ & .255 & $.307 x$ & .219 & .180 & .209 & .208 \\
\hline LM+WE $(\lambda=0.3)$ & .205 & $.281 y$ & .198 & .107 & .167 & .154 \\
\hline LM+WE $(\lambda=0.5)$ & .236 & $.299 x$ & .215 & .123 & .203 & .183 \\
\hline LM+WE $(\lambda=0.7)$ & .286 & $.317 x$ & .222 & .190 & .249 & .225 \\
\hline
\end{tabular}




\section{Results - CLIR}

\begin{tabular}{|c|c|c|c|c|c|c|}
\hline \multirow[b]{2}{*}{ Model } & \multicolumn{3}{|c|}{$\mathrm{NL} \rightarrow \mathrm{EN}$} & \multicolumn{3}{|c|}{$\mathrm{EN} \rightarrow \mathrm{NL}$} \\
\hline & 2001 & 2002 & 2003 & 2001 & 2002 & 2003 \\
\hline LM-UNI & .094 & .108 & .092 & .078 & .125 & .112 \\
\hline $\begin{array}{l}\text { LDA-IR } \\
\operatorname{dim}: 300 ; c s: 60\end{array}$ & .197 & .139 & .123 & .145 & .137 & .171 \\
\hline $\begin{array}{l}\text { WE-VS } \\
\operatorname{dim}: 600 ; c s: 60\end{array}$ & .187 & $.204 x$ & .120 & .174 & $.185 y$ & .157 \\
\hline WE-VS & $.222 y$ & $.230 x$ & .127 & $.178 y$ & $.219 x$ & .181 \\
\hline LM+LDA & .267 & .225 & .199 & .225 & .268 & .278 \\
\hline $\begin{array}{l}\mathrm{GT}+\mathrm{LM}+\mathrm{LDA} \\
\operatorname{dim}: 300 ; c s: 60\end{array}$ & .307 & .275 & .248 & .230 & .240 & .244 \\
\hline LM+WE $(\lambda=0.3)$ & .189 & .273 & .197 & .101 & .159 & .150 \\
\hline LM+WE $(\lambda=0.5)$ & .218 & $.283 y$ & .220 & .113 & .184 & .167 \\
\hline $\begin{array}{l}\text { LM+WE }(\lambda=0.7) \\
\operatorname{dim}: 600 ; c s: 60\end{array}$ & .255 & $.307 x$ & .219 & .180 & .209 & .208 \\
\hline LM+WE $(\lambda=0.3)$ & .205 & $.281 y$ & .198 & .107 & .167 & .154 \\
\hline LM+WE $(\lambda=0.5)$ & .236 & $.299 x$ & .215 & .123 & .203 & .183 \\
\hline LM+WE $(\lambda=0.7)$ & .286 & $.317 x$ & .222 & .190 & .249 & .225 \\
\hline
\end{tabular}




\section{Results - CLIR}

\begin{tabular}{|c|c|c|c|c|c|c|}
\hline \multirow[b]{2}{*}{ Model } & \multicolumn{3}{|c|}{$\mathrm{NL} \rightarrow \mathrm{EN}$} & \multicolumn{3}{|c|}{$\mathrm{EN} \rightarrow \mathrm{NL}$} \\
\hline & 2001 & 2002 & 2003 & 2001 & 2002 & 2003 \\
\hline LM-UNI & .094 & .108 & .092 & .078 & .125 & .112 \\
\hline $\begin{array}{l}\text { LDA-IR } \\
\text { dim: } 300 ; c s: 60\end{array}$ & .197 & .139 & .123 & .145 & .137 & .171 \\
\hline $\begin{array}{l}\text { WE-VS } \\
\operatorname{dim}: 600 ; c s: 60\end{array}$ & .187 & $.204 x$ & .120 & .174 & $.185 y$ & .157 \\
\hline WE-VS & $.222 y$ & $.230 x$ & .127 & $.178 y$ & $.219 x$ & .181 \\
\hline LM+LDA & .267 & .225 & .199 & .225 & .268 & .278 \\
\hline $\begin{array}{l}\text { GT+LM+LDA } \\
\operatorname{dim}: 300 ; c s: 60\end{array}$ & .307 & .275 & .248 & .230 & .240 & .244 \\
\hline LM+WE $(\lambda=0.3)$ & .189 & .273 & .197 & .101 & .159 & .150 \\
\hline LM+WE $(\lambda=0.5)$ & .218 & $.283 y$ & .220 & .113 & .184 & .167 \\
\hline $\begin{array}{l}\text { LM+WE }(\lambda=0.7) \\
\text { dim: } 600 ; c s: 60\end{array}$ & .255 & $.307 x$ & .219 & .180 & .209 & .208 \\
\hline LM+WE $(\lambda=0.3)$ & .205 & $.281 y$ & .198 & .107 & .167 & .154 \\
\hline $\mathrm{LM}+\mathrm{WE}(\lambda=0.5)$ & .236 & $.299 x$ & .215 & .123 & .203 & .183 \\
\hline LM+WE $(\lambda=0.7)$ & .286 & $.317 x$ & .222 & .190 & .249 & .225 \\
\hline
\end{tabular}




\section{Results - CLIR II}

\begin{tabular}{|c|c|c|c|c|c|c|}
\hline \multirow[b]{2}{*}{ Model } & \multicolumn{3}{|c|}{$\mathrm{NL} \rightarrow \mathrm{EN}$} & \multicolumn{3}{|c|}{$\mathrm{EN} \rightarrow \mathrm{NL}$} \\
\hline & 2001 & 2002 & 2003 & 2001 & 2002 & 2003 \\
\hline LM+LDA & .267 & .225 & .199 & .225 & .268 & .278 \\
\hline $\mathrm{GT}+\mathrm{LM}+\mathrm{LDA}$ & .307 & .275 & .248 & .230 & .240 & .244 \\
\hline \multicolumn{7}{|l|}{$\operatorname{dim}: 600 ; c s: 60$} \\
\hline $\operatorname{LM}+\mathrm{WE}(\lambda=0.3)$ & .205 & $.281 y$ & .198 & .107 & .167 & .154 \\
\hline LM+WE $(\lambda=0.5)$ & .236 & $.299 x$ & .215 & .123 & .203 & .183 \\
\hline LM+WE $(\lambda=0.7)$ & .286 & $.317 x$ & .222 & .190 & .249 & .225 \\
\hline \multicolumn{7}{|l|}{$\operatorname{dim}: 600 ; c s: 60$} \\
\hline LM+LDA+WE $(\lambda=0.3)$ & .277 & .263 & .210 & .229 & .288 & .283 \\
\hline LM+LDA+WE $(\lambda=0.5)$ & $.281 y$ & $.281 y$ & .214 & .240 & $.297 y$ & .290 \\
\hline LM+LDA+WE $(\lambda=0.7)$ & $.302 x$ & $.302 x$ & .227 & $.244 y$ & $.311 x$ & $.302 y$ \\
\hline
\end{tabular}




\section{Results - Composition}

\begin{tabular}{|c|c|c|c|c|c|c|}
\hline \multirow[b]{3}{*}{ Composition } & \multicolumn{6}{|c|}{ Monolingual } \\
\hline & \multicolumn{3}{|c|}{$\mathrm{EN} \rightarrow \mathrm{EN}$} & \multicolumn{3}{|c|}{$\mathrm{NL} \rightarrow \mathrm{NL}$} \\
\hline & 2001 & 2002 & 2003 & 2001 & 2002 & 2003 \\
\hline ADD-BASIC (300-60) & .324 & .258 & .257 & .203 & .237 & .224 \\
\hline ADD-SI (300-60) & .338 & $.278 y$ & .255 & .212 & $.253 y$ & .227 \\
\hline ADD-BASIC (600-60) & .329 & .281 & .262 & .204 & .262 & .231 \\
\hline ADD-SI (600-60) & $.344 y$ & $.301 y$ & .263 & .215 & $.275 y$ & .234 \\
\hline
\end{tabular}




\section{Results - Composition II}

\begin{tabular}{|c|c|c|c|c|c|c|}
\hline \multirow[b]{3}{*}{ Composition } & \multicolumn{6}{|c|}{ Cross-lingual } \\
\hline & \multicolumn{3}{|c|}{$\mathrm{NL} \rightarrow \mathrm{EN}$} & \multicolumn{3}{|c|}{$\mathrm{EN} \rightarrow \mathrm{NL}$} \\
\hline & 2001 & 2002 & 2003 & 2001 & 2002 & 2003 \\
\hline ADD-BASIC (300-60) & .187 & .204 & .120 & .174 & .185 & .157 \\
\hline ADD-SI (300-60) & $.216 x$ & $.213 y$ & .122 & $.189 y$ & $.208 x$ & .161 \\
\hline ADD-BASIC (600-60) & .221 & .230 & .127 & .178 & .219 & .181 \\
\hline ADD-SI (600-60) & $.237 y$ & .233 & .130 & .189 & $.229 x$ & .184 \\
\hline
\end{tabular}




\section{Summary of Contributions (Repeated)}

- A novel model for learning bilingual word embeddings (BWEs) from non-parallel document-aligned data

- A simple framework for constructing query and document embeddings

- A unified framework for MolR and CLIR based on (bilingual) word embeddings 


\section{So, what's next? I}

The proposed framework is very general:

Designing other shuffling procedures for BWESG

Building new BWE induction models for the same multilingual data type (remove the need for pseudo-bilingual documents?

Experimenting with other monolingual WE induction models for BWESG besides SGNS

Investigating other BWE induction models (different bilingual signals) in the same (CL)IR pipeline 


\section{So, what's next? II}

Investigating more elaborate composition models to construct document and query embeddings (what about syntax?)

Testing true paragraph and phrase embeddings in the same $(C L) I R$ pipeline

[Le and Mikolov, ICML 2014; Soyer et al., ICLR 2015]

Other (more distant) language pairs, other queries+test collections

Combining the semantic BWE-based knowledge with other IR modeling paradigms (besides the ones mentioned here) 


\section{Questions?}
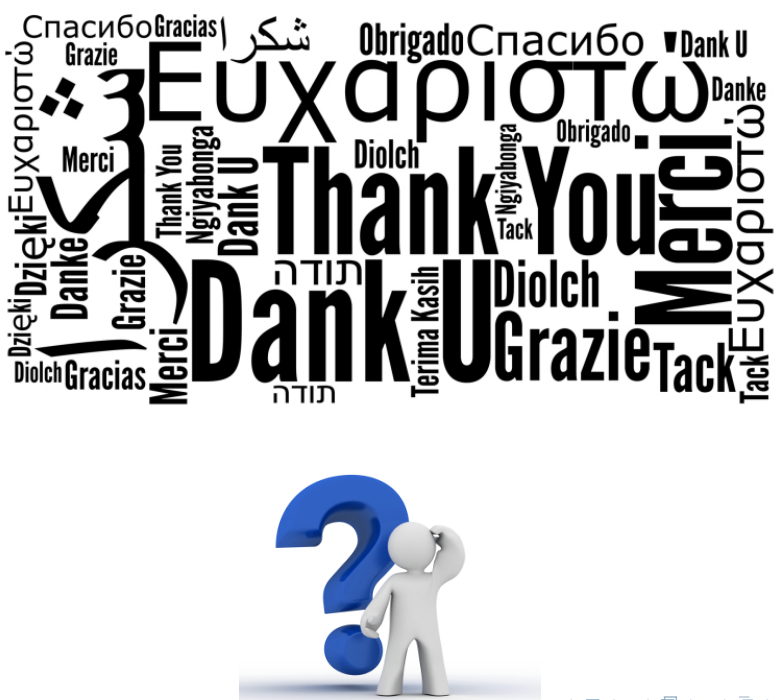